\title{
Reply to the correspondence letter: "Hyperbilirubinemia in breastfed term neonates"
}

\author{
Ying-Juang Chen • Wei-Chuan Chen • \\ Chung-Ming Chen
}

Received: 5 December 2011 / Accepted: 9 December 2011 /Published online: 22 December 2011

(C) Springer-Verlag 2011

\section{Dear Editor,}

We would like to thank Dr. Das for his comments on our article [2]. The incidence of neonatal hyperbilirubinemia is different among races. Exclusive breastfeeding and East Asian ethnicity are major risk factors for the development of hyperbilirubinemia in term infants [1]. The incidence of hyperbilirubinemia in our study is comparable to another study that was also conducted in Taiwanese neonates [3]. Blood group incompatibility, G6PD deficiency, previous sibling received phototherapy, male sex, infant of diabetic mother, and East Asian race are risk factors for hyperbilirubinemia in term infants. Our study population is Taiwanese neonates who were exclusively breastfed without formula supplementation at any time or before developing hyperbilirubinemia. We found that gender was similar between the hyperbilirubinemic and non-hyperbilirubinemic groups, and mean hemoglobin was $15.8 \mathrm{~g} / \mathrm{dl}$ in the hyperbilirubinemic and readmitted neonates. Neonatal birth trauma was more frequent in a vaginal delivery than in a cesarean section [5]. Birth trauma was not a significant risk factor for hyperbilirubinemia because we found that a significantly higher percentage of neonates were delivered via cesarean section in the hyperbilirubinemic neonates. However, we did not record the prevalence of previous sibling received phototherapy and infant of diabetic mother.

A length of stay of $<72 \mathrm{~h}$ is a risk factor for readmission with hyperbilirubinemia [4]. The American Academy of Pediatrics recommends closer follow-up for infants discharged $<48 \mathrm{~h}$ of age. Mean age at nursery discharge and mean age at

Y.-J. Chen · W.-C. Chen · C.-M. Chen $(\triangle)$

Department of Pediatrics, Taipei Medical University Hospital,

Taipei, Taiwan

e-mail: cmchen@tmu.edu.tw

\section{C.-M. Chen}

Department of Pediatrics, School of Medicine, College of Medicine, Taipei Medical University,

Taipei, Taiwan outpatient department follow-up was 3.2 days and 6 days, respectively, in our study population. Their length of stay was $>72 \mathrm{~h}$ and was seen by a health care professional within 2 to 3 days of discharge. Therefore, we suggest that the length of stay in the nursery is not a major risk factor for hyperbilirubinemia in exclusively breastfed infants in our study.

Cesarean section was reported to protect newborn infants against developing neonatal jaundice. The exact causes of this protective effect were unclear. Our study found that a slightly higher percentage of hyperbilirubinemic neonates had been delivered via cesarean section. We speculated that loss of protective effects of cesarean section may be due to our hospital being in a room-in setting and all healthy newborns are initiated into breastfeeding in the delivery room and were breastfed on demand irrespective of delivery route.

We agree with Dr. Das that risk factors for jaundice in breastfed infants need to be clearly defined. Understanding these risk factors will guide clinicians in planning appropriate follow-up strategies for neonates discharged from the nursery with jaundice.

Conflict of interest The authors have no conflict of interest.

\section{References}

1. American Academy of Pediatrics (2004) Management of hyperbilirubinemia in the newborn infant 35 or more weeks of gestation. Pediatrics 114:297-316

2. Chen YJ, Chen WC, Chen CM (2011) Risk factors for hyperbilirubinemia in breastfed term neonates. Eur J Pediatr. doi:10.1007/ s00431-011-1512-8

3. Chou HC, Chen MH, Yang HI et al (2011) $211 \mathrm{G}$ to A variation of UDP-glucuronosyl transferase 1A1 gene and neonatal breastfeeding jaundice. Pediatr Res 69:170-174

4. Maisels MJ, Kring EA (1998) Length of stay, jaundice, and hospital readmission. Pediatrics 101:995-998

5. Mosavat SA, Zamani M (2008) The incidence of birth trauma among live born term neonates at a referral hospital in Rafsanjan, Iran. J Matern Fetal Neonatal Med 21:337-339 